\title{
Real world experience with dose dense ac-paclitaxel: Two canadian cancer centers' experience
}

\author{
Emad Tashkandi ${ }^{1}$, Michael Yan ${ }^{2}$, J awaid Younus ${ }^{3}$, MA Jawaid ${ }^{4}$, Caroline Hamm ${ }^{5}$, Swati Kulkarni ${ }^{5}$, \\ Rasna Gupta ${ }^{5}$, J ohn Matthews ${ }^{5}$, Tarek Elfiki ${ }^{5}$, Larry Stitt ${ }^{6}$ \\ 1. Department of Medical Oncology, McMaster University, Canada. 2. Schulich School of Medicine and Dentistry, Canada. \\ 3. London Regional Cancer Program, Canada. 4. Western University Social Sciences, Canada. 5. Windsor Regional Cancer \\ Centre, Canada. 6. Clinical Research Unit, London Regional Cancer Program, Canada.
}

Correspondence: Dr. Jawaid Younus. Address: 790 Commissioners Road East London, ON, N6A 4L6, Canada. Email: jawaid.younus@Ihsc.on.ca

Received: April 29, $2015 \quad$ Accepted: May 25, $2015 \quad$ Online Published: July 13, 2015

DOI : 10.5430/jst.v5n2p86 URL: http://dx.doi.org/10.5430/jst.v5n2p86

\section{Abstract}

Early breast cancer treatment with dose dense Adriamycin-Cyclophosphamide and Paclitaxel (AC-P) has been shown to increase survival. However, it is commonly associated with neutropenia, anemia or both.

This retrospective chart review study was done to evaluate the real world experience with this regimen and included a series of 83 adult women from the London Regional Cancer Program and 50 patients from the Windsor Regional Cancer Center who were treated with dose dense adjuvant AC-P for early breast cancer from January 2009 to August 2012. Toxicities like febrile neutropenia (FN) and anemia based on NCIC-CTC v2 criteria and grades were recorded along with the use of erythropoietin stimulating agents (ESA), Neupogen or Neulasta, and blood transfusion.

The majority of our patients (88.72\%) were able to complete all 8 cycles of AC-Taxol, although 32 of these patients (24.06\%) experienced delay during their treatment. Grade 3 anemia was seen in one patient after cycle \#4 and increased to two patients after both cycles 5 and 7. Only one patient developed grade 4 anemia, observed in the 5th cycle. Blood transfusion was given to sixteen patients and three patients received ESA. The incidence of febrile neutropenia was only $3.00 \%$.

The majority of our patients completed all 8 cycles of dose dense AC-Paclitaxel without any delay. The use of Neupogen and Neulasta effectively kept the incidence of FN to $3.00 \%$ and only a minority of patients experienced anemia requiring transfusion or ESA. Based on our experience, the dose-dense schedule with AC-Paclitaxel is a feasible and tolerable regimen to treat patients with early breast cancer.

\section{Key words}

Dose dense chemotherapy, Adriamycin-cyclophosphamide, Paclitaxel, Febrile neutropenia, Anemia

\section{I ntroduction}

Breast cancer is one of the most common cancers to affect women annually on a global scale. In 2014, the estimated number of new cases of female breast cancer in Canada was 24,200, with 1 in 30 chance of dying from this disease ${ }^{[1]}$. 
For primary breast cancer, the standard of care involves surgical resection and may require radiation therapy and/or chemotherapy. Among chemotherapeutic agents, anthracycline-containing polychemotherapy regimens are the mainstay of treatment. Compared to the traditional cyclophosphamide-methotrexate-5 fluorouracil (CMF) regimen, anthracyclinecontaining treatments such as Adriamycin-Cyclophosphamide (AC) or Epirubicin-Cyclophosphamide (EC) have been shown to be more effective in reducing recurrence and overall mortality rates with annual hazard ratio reductions of $23.5 \%$ and $15.3 \%$ respectively ${ }^{[2]}$. The addition of taxanes to anthracycline-containing adjuvant chemotherapy have shown improvements in disease free survival and possibly even mortality rates ${ }^{[3,4]}$.

However, these chemotherapy regimens are associated with significant toxicity. To address this issue, treatment schedules involve cycling between treatment and recovery periods in order to allow patients to recuperate from the damages induced by the chemotherapy. The concern with allowing for recovery time is that the remaining cancer cells are able to rehabilitate and potentially divide during this period, increasing the chance of recurrence in future. Hence the concept of dose density has garnered much recent attention. Instead of dose escalation, dose dense regimens are able to increase treatment intensity by minimizing the duration of the recovery period and effectively increasing the density of treatment frequency ${ }^{[5]}$. Trials comparing the efficacy of dose dense versus conventional regimens have shown promising results in reducing cancer recurrence as well as overall mortality ${ }^{[6-10]}$. In particular, the large phase III CALBG C9741 trial has shown risk reductions of $26 \%$ and $31 \%$ for cancer recurrence and mortality respectively ${ }^{[6]}$.

With such dose intensity, treatment toxicities increase as well. Adriamycin-containing chemotherapy regimens potentiate numerous toxicities that affect the gastrointestinal, musculoskeletal, cardiac systems, and in particular the hematological system. Current evidence however does not appear to support an increase in treatment adverse events compared to conventional scheduled therapy ${ }^{[6,11-14]}$. This is largely due in part to the use of granulocyte colony stimulating factor (G-CSF) supplementation as a primary prophylaxis to prevent the occurrence of treatment-induced neutropenia.

Our study investigates the incidence of major hematological toxicities associated with the dose dense AC-paclitaxel regimen and the effects of these toxicities on schedule adherence and supplementary treatment necessitation, as a real world experience.

\section{Methods}

\subsection{Patient selection criteria}

Patients were eligible for this retrospective study if they had a confirmed diagnosis of invasive mammary carcinoma and had been recommended to have adjuvant chemotherapy that included 8 cycles of dose dense AC-Paclitaxel biweekly for a total of 16 weeks. Adriamycin and Cytoxan were to be administered for the first 4 cycles followed by Paclitaxel for the last 4 cycles. All patients were also to have received concurrent G-CSF treatment with Neupogen or Neulasta as a primary prophylaxis (see Figure 1).

Figure 1. Treatment Outline. Patients from London and Windsor with early breast cancer were treated with dose dense AC-Paclitaxel regimen. Adriamycin and Cyclophosphamide were administered once every two weeks for four cycles, followed up with biweekly Paclitaxel treatment for another four cycles. Treatment was supplemented with G-CSF administration at the beginning of the regimen.

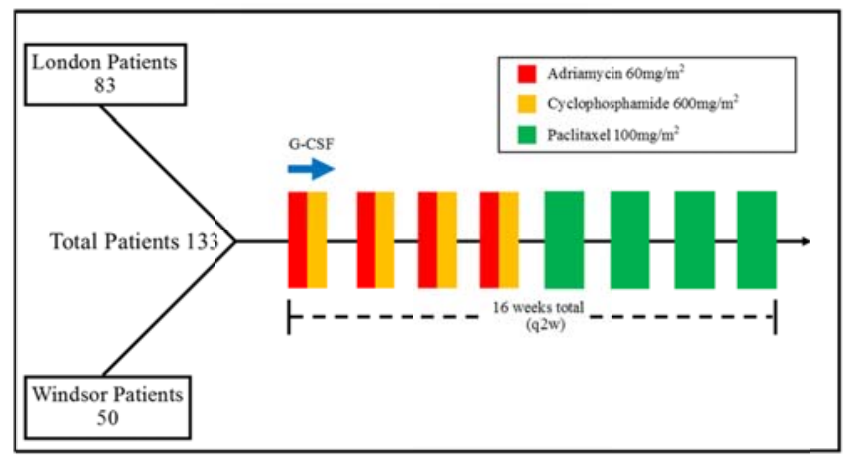


This retrospective review study was approved by the ethics committee at the University of Western Ontario.

\subsection{Baseline criteria}

Patient parameters at baseline were collected and included age, weight, ER/PR/Her2-Neu status, and tumor staging (see Table 1). Bloodwork was done prior to treatment initiation as well as prior to the start of each of the 8 cycles. Complete blood counts and differentials were performed focusing specifically on hemoglobin value, white blood cell value, and absolute neutrophil count in order to monitor primary chemotherapy toxicity endpoints.

Table 1. Receptor Status and Tumor Staging

\begin{tabular}{lll}
\hline Characteristic & Number of Patients & Percentage (\%) \\
\hline Total Cohort & 133 & 100 \\
London & 83 & 62.41 \\
Windsor & 50 & 37.59 \\
Receptor Status & & \\
ER & 77 & 57.89 \\
Positive & 56 & 42.11 \\
Negative & & \\
PR & 67 & 50.38 \\
Positive & 66 & 49.62 \\
Negative & & \\
Her2/Neu & 26 & 79.70 \\
Positive & 106 & 19.55 \\
Negative & 1 & 0.75 \\
Equivocal & & \\
TNM Staging & 21 & 15.79 \\
Stage 1 & 76 & 57.14 \\
Stage 2 & 35 & 26.32 \\
Stage 3 & 1 & 0.75 \\
Stage 4 & &
\end{tabular}

\subsection{Treatment}

All patients received dose dense chemotherapy with $60 \mathrm{mg} / \mathrm{m}^{2}$ Adriamycin and $600 \mathrm{mg} / \mathrm{m}^{2}$ Cyclophosphamide IV once biweekly for 4 doses. Subsequently $175 \mathrm{mg} / \mathrm{m}^{2}$ Paclitaxel was administered once biweekly for another 4 doses. All patients receiving chemotherapy were supplemented with granulocyte-colony stimulating factor. The dose of Neupogen used was $300 \mathrm{mcg}$ or $480 \mathrm{mcg}$ based according to body weight, mostly for 10 days per cycle while Neulasta was administered as a single dose of $6 \mathrm{mg}$ after each cycle of chemotherapy. A minority of patients switched between G-CSF supplements and resultantly received both in a sequential manner. Clinicians, in accordance to their usual clinical practice, also ordered packed red blood cell transfusions and erythropoietin stimulating agent (Darbepoetin Alfa or Epoetin Alfa) administration as needed (see Figure 2).

Figure 2. G-CSF Administration Outline. All but one of 133 patients were supplemented with G-CSF at the start of their dose dense chemotherapy regimen. Patients were given either Neupogen or Neulasta, Some patients switched between the two and as a result received both. Patients given Neupogen either received a $300 \mathrm{mcg}$ or $480 \mathrm{mcg}$ dose.

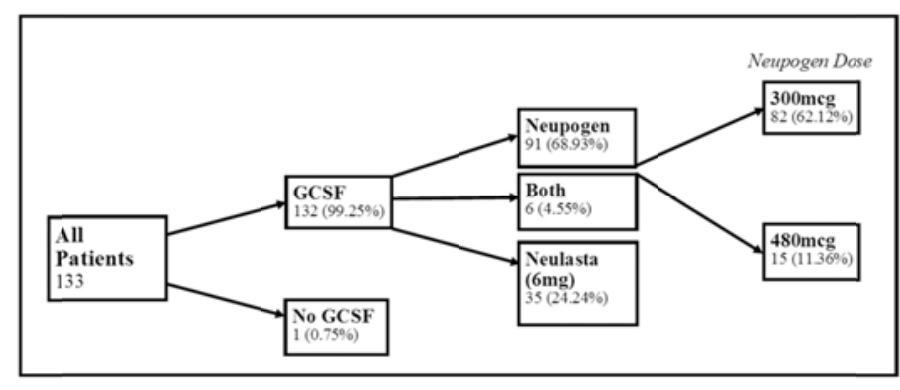




\subsection{Toxicity assessment}

The primary objective of this retrospective study is to observe the incidence of toxic events, including anemia and febrile neutropenia according to NCIC Common Toxicity Criteria Version 215. Secondary objectives include the incidence of non-anemic or neutropenic toxicity mandated chemotherapy schedule delays, blood transfusion requirements, and ESA administration needs. If chemotherapy delay occurred, the physician's rational as well as cycle number were recorded.

\section{Results}

\subsection{Patients}

Between January 2009 and August 2012, 83 female patients from the London Regional Cancer Program and the 50 patients from Windsor Regional Cancer Centre with early breast cancer were entered into this study as a single cohort of 133 patients. Only one patient with questionable bone metastases was considered as stage IV disease in this cohort and is included based on the approach as intent to treat analysis for this study. Median patient age was 53 years (range, 18-84 years) and median weight was $74 \mathrm{~kg}$ (range, 48-131 kg). Patients' baseline characteristics including hormone receptor status as well as tumor staging can be seen in Table 1.

All but one of the 133 patients received some form of G-CSF treatment (99.25\%), with Neupogen administered to 91 patients (68.94\%), Neulasta to 35 patients (26.32\%). Six patients (4.51\%) received both Neupogen and Neulasta during the course of their treatment. Of the patients who received Neupogen, 82 (61.65\%) were put on the 300 mcg dose while the remaining 15 received a $480 \mathrm{mcg}$ dose $(11.28 \%)$. Apart from dosage, the administration schedule also varied with 88 patients (66.17\%) receiving 10 days of Neupogen per treatment cycle, seven patients (5.26\%) received Neupogen 7 days per cycle, while the 5 and 8 day regimens each had one patient $(0.75 \%)$.

\subsection{Toxicity}

Grade 2 or greater anemia events occurred 119 times over the 8 cycles. Grade 2 hemoglobin values were first observed in one patient (0.75\%) in cycle 2 and increased in cycle 3 to three patients (2.26\%) all the way to cycles 4 and 5 with 18 (13.74\%) and 30 patients (22.73\%) respectively. The number of grade 2 anemic patients then declined slightly in cycle 6 with 20 patients (16.13\%), rose up in cycle 7 to 26 patients (21.31\%), before dropping back down to 15 patients (12.93\%) in cycle 8 . Grade 3 anemia was seen in one patient (0.76\%) after cycle 4 and in two patients in each cycle 5 (1.52\%) and 7 (1.64\%). Only one patient (0.76\%) developed grade 4 anemia, observed in the 5th cycle.

Grade 2 or higher neutropenia occurred 26 times throughout the duration of the treatment cycles, with the grade 2 neutropenia initially observed in two patients (1.50\%) in cycle 2. This value increased to 3 patients $(2.26 \%)$ at the end of cycle 3 and then to 5 patients (3.82\%) at the end of cycle 4 before dropping back down to 2 patients (1.52\%) at the end of cycle 5 . Three patients (2.42\%) were observed in cycle 6, one patient (0.82\%) in cycle 7 , and three patients (2.61\%) were observed with grade 2 neutropenia at the end of cycle 8. Grade 3 neutropenia was observed in one patient at the end of cycles $3(0.75 \%)$ and $8(0.87 \%)$. We observed grade 4 neutropenia in cycle 3 in three patients $(2.26 \%)$, as well as in cycles 4 and 5 in one patient $(0.76 \%)$ for each cycle.

Of the 133 patients in the trial, 3.00\% of patients experienced febrile neutropenia (FN). FN was observed in four of the 133 patients, occurring in two patients (1.50\%) at the end of cycle 2, and then one patient (0.75\%) each at the end of cycles 3 and 4. Pre-treatment ANC values for subsequent chemotherapy cycles ranged from 0.3-0.8.

The occurrences of primary toxicity endpoints are listed in Table 2, with Table 2B showing specifically the details of the febrile neutropenic events. 
Table 2A. Primary Toxicity Endpoints

\begin{tabular}{|c|c|c|c|c|c|c|c|c|c|}
\hline Toxicity & \multicolumn{8}{|c|}{$\begin{array}{c}\text { Number of Occurrences (\%) } \\
\text { Cycle Number }\end{array}$} & \multirow[t]{2}{*}{$\begin{array}{l}\text { Total } \\
\text { occurrence }\end{array}$} \\
\hline \multicolumn{9}{|c|}{ Neutropenia } & \\
\hline Grade & 1 & 2 & 3 & 4 & 5 & 6 & 7 & 8 & \\
\hline 1 & $3(2.26)$ & $5(3.76)$ & $3(2.26)$ & $1(0.76)$ & $3(2.27)$ & - & $1(0.82)$ & $1(0.87)$ & 17 \\
\hline 2 & - & $2(1.50)$ & $3(2.26)$ & $5(3.82)$ & $2(1.52)$ & $3(2.42)$ & $1(0.82)$ & 3 (2.61) & 19 \\
\hline 3 & - & - & $1(0.75)$ & - & - & - & - & $1(0.87)$ & 2 \\
\hline 4 & - & - & 3 (2.26) & $1(0.76)$ & $1(0.76)$ & - & - & - & 5 \\
\hline \multicolumn{10}{|c|}{$\begin{array}{l}\text { Febrile } \\
\text { Neutropenia }\end{array}$} \\
\hline \multicolumn{9}{|l|}{ Anemia } & 4 \\
\hline \multicolumn{10}{|l|}{ Grade } \\
\hline 1 & $3(2.26)$ & $10(7.52)$ & 26(19.55) & $36(27.48)$ & 51(38.64) & 48(38.71) & 43(35.25) & ) & 261 \\
\hline 2 & - & $1(0.75)$ & $3(2.26)$ & 18 (13.74) & 30 (22.73) & 20 (16.13) & $26(21.31)$ & $\begin{array}{l}15 \\
(12.93)\end{array}$ & 113 \\
\hline 3 & - & - & - & $1(0.76)$ & $2(1.52)$ & - & $2(1.64)$ & - & 5 \\
\hline 4 & - & - & - & - & $1(0.76)$ & - & - & - & 1 \\
\hline $\begin{array}{l}\text { Patients } \\
\text { Missing }\end{array}$ & 0 & 0 & 0 & 2 & 1 & 9 & 11 & $\begin{array}{l}18 \mathrm{~N} / 17 \\
\mathrm{~A}\end{array}$ & \\
\hline
\end{tabular}

Table 2B. Febrile Neutropenia Events

\begin{tabular}{llll}
\hline Age & Cycle Number & WBC Value & ANC Value \\
\hline 44 & 4 & 8.9 & $5.9^{*}$ \\
60 & 2 & 0.8 & 0.4 \\
65 & 3 & 0.8 & 0.4 \\
71 & 2 & 0.5 & 0.5 \\
\hline
\end{tabular}

*Note that for this patient, the febrile neutropenic event occurred one week post cycle 4 , where ANC values dropped as low as 0.3 , and patient experienced a fever of $37.8^{\circ} \mathrm{C}$.

\subsection{Treatment delays}

Out of 133 patients, 118 (88.72\%) were able to complete all 8 cycles of the treatment regimen (see Table 3A). In total 32 patients (24.06\%) experienced some delay during their treatment. Delays were first observed at the end of cycle 2 in two patients and increased as the cycles progressed with four patients in cycle 3, five patients in cycle 4, and peaking at cycles 5 and 6, each having seven patients who experienced delay. Delay frequency then dropped back down to four patients in cycle 7 and then to three patients experiencing delay during cycle 8 (see Table 3B).

Table 3A. Cycles Completed

\begin{tabular}{ll}
\hline Cycle Completed & Incidence (\%) \\
\hline Cycle Number & \\
3 & $1(0.75)$ \\
4 & - \\
5 & $7(5.26)$ \\
6 & $2(1.50)$ \\
7 & $5(3.76)$ \\
8 & $118(88.72)$ \\
\hline
\end{tabular}

Table 3B. Delay in Cycle

\begin{tabular}{ll}
\hline Cycle Completed & Incidence (\%) \\
\hline Cycle Number & \\
2 & $2(1.50)$ \\
3 & $4(3.00)$ \\
4 & $5(3.78)$ \\
5 & $7(5.38)$ \\
6 & $7(5.60)$ \\
7 & $4(3.25)$ \\
8 & $3(2.54)$ \\
\hline
\end{tabular}


Table 3C. Reason for Delay

\begin{tabular}{ll}
\hline Reason & Incidence (\%) \\
\hline Blood Counts & $3(2.26)$ \\
Patient Choice & $1(0.75)$ \\
Toxicity & $3(2.26)$ \\
Anemia & $2(1.50)$ \\
Febrile Neutropenia & $1(0.75)$ \\
Peripheral Neuropathy & $2(1.50)$ \\
Thrombocytopenia & $1(0.75)$ \\
Neutropenia & $1(0.75)$ \\
Nausea and Vomiting & $4(3.00)$ \\
Skin Rash & $2(1.50)$ \\
Multiple Toxicities & $2(1.50)$ \\
Fevers & $2(1.50)$ \\
Lower Limb Pain & $1(0.75)$ \\
Febrile Neutropenia and RTI & $2(1.50)$ \\
Respiratory Symptoms & $1(0.75)$ \\
Skin rash + Dyspnea & $1(0.75)$ \\
Dysphagia + Rash & \\
Hospitalization Due to Toxicity & $1(0.75)$ \\
Pneumonia & $1(0.75)$ \\
Dyspnea & $1(0.75)$ \\
Hospitalization due to Other Condition &
\end{tabular}

The reasons for treatment delay were numerous, and included abnormal blood count, patient choice, surgeryhospitalization, fever, and various other toxicities (see Table 3C). The most common reason for treatment delay was the presentation of skin rash indicating toxicity, which was observed in four patients. Low neutrophil counts as well as anemia were the next most common reasons for treatment delay, with three patients having either toxicity. Febrile neutropenia, thrombocytopenia, fever, and respiratory symptoms were observed with two patients in each toxicity category. Only two patients required hospitalization were recorded, with one due to dyspnea and the other due to pneumonia.

\subsection{Blood transfusions and ESA administration}

Sixteen of the 133 patients (11.27\%) required blood transfusions for anemia treatment. The majority, 14 patients (10.53\%), received 2 units of packed red blood cells while only two patients (1.50\%) got transfused with 4 units. Three patients in total were given ESA treatment. Two patients were treated with Darbepoetin Alfa while Epoetin Alfa was given to only one patient.

The average pretreatment hemoglobin value was $87.7 \mathrm{~g} / \mathrm{L}$, with a range from 70-117 g/L. The average cycle number necessitating intervention was 5.6, with a range of cycle 4-8 (see Table 4).

Table 4. ESA and Transfusion Requirements for Anemic Patients

\begin{tabular}{llllll}
\hline Procedure & Incidence (\%) & HgB Average (g/L) & HgB Range (g/L) & Cycle Average & Cycle Range \\
\hline ESA & $2(1.50)$ & & & \\
Darbepoetin Alfa & $1(0.75)$ & & & \\
Epoetin Alfa & & $70-117$ & \\
Packed RBC Transfusion & $14(10.53)$ & & & \\
2 units & $2(1.50)$ & & & \\
4 units & & & \\
\hline
\end{tabular}




\section{Discussion}

Our retrospective chart review showed that the dose dense AC-Paclitaxel therapy produced a low incidence of serious primary endpoint toxicities, allowing the majority of our patients (88.72\%) to complete all 8 cycles of the regimen without delay. Of the minority of patients that did require treatment delay, most were due to hematological reasons. With primary prophylactic G-CSF supplementation, only 5.26\% of all treatment delays were caused by neutropenic toxicities. Other reasons for delay included skin rashes, fevers, and respiratory difficulties. Only two patients in this cohort required hospitalization during their treatment.

Overall dose dense therapy results did not suggest increased toxicities compared to results reported by conventional AC-Paclitaxel therapy, such as those from trial NSABP B-283. NSABP B-28 had almost 76\% patients able to complete all 8 cycles of therapy and the FN incidence was observed to be 3.0\%. Likewise, the hallmark CALBG C9741 trial that compared dose dense AC-paclitaxel therapy with sequential and conventional treatments found grade 3+ neutropenia better in dose-dense regimen patients in comparison to conventionally treated patients at $6 \%$ and $12 \%$ incidences respectively. This is attributed to the primary prophylaxis with GCSF in the dose dense arm. Hospitalizations due to FN were $2 \%$ in dose dense patients compared to $6 \%$ in the conventional cohort. Grade 4 hemoglobin toxicity differences were negligible, with a $1 \%$ dose dense cohort incidence compared to nil patients undergoing conventional treatment. In terms of treatment delay, the incidence was $31 \%$ in the dose dense cohort compared to $39 \%$ in the conventional treatment group. Overall, this study suggested that dose dense scheduling was superior to the conventional regimen in regards to treatment toxicity amongst other endpoints ${ }^{[6]}$.

Although grade 2 and 3 anemias were frequent in our study, only 16 patients (12.03\%) were given blood transfusions, in addition to the three patients treated with ESA. The CALBG C9741 study reported 3\% of dose dense patients and $4 \%$ of conventional patients requiring packed RBC transfusion ${ }^{[6]}$. The decision to transfuse packed red blood cells depends on several factors including the hemoglobin value, age, comorbid condition and symptoms. This may explain the discrepancy of lower incidence of PRBC transfusions compared to a higher number of grade ${ }^{[2-4]}$ anemia observed in our cohort.

Further insight in to the safety of dose dense chemotherapy regimens can be found in two European studies that compared standard versus dose dense epirubicin, cyclophosphamide, and 5-fluorouracil (ECF) chemotherapy. Baldini et al. ${ }^{[13]}$ concluded that chemotherapy-related toxicities were mild and superimposable between arms, with $18.9 \%$ and $23.5 \%$ of patients experiencing neutropenia in the conventional and dose dense arms respectively. There was no incidence of febrile

neutropenia in either cohorts ${ }^{[13]}$. Venturini et al. ${ }^{[14]}$ found that patients in the dose dense cohort had a lower incidence of treatment delays and dose reductions (26\% compared to 33\%) as well as a $33 \%$ risk reduction of leukopenic events. Certain toxicities however were notably increased in the dose dense arm, including anemia, asthenia, bone pain, and thrombocytopenia ${ }^{[14]}$.

\section{Conclusion}

The dose dense chemotherapy is commonly used as an adjuvant treatment for early breast cancer. Based on the results of our study, we found that from a tolerability and toxicity end point, real world experience from two Canadian cancer centres matches with the results recorded in randomized clinical trials for dose dense regimens.

\section{Conflict of interests}

The authors declare that they have no competing interests.

\section{References}

[1] 2014 Canadian Cancer Statistics, Govt. of Canada:

http://www.cancer.ca/ /media/cancer.ca/CW/cancer\%20information/cancer\%20101/Canadian\%20cancer\%20statistics

/Canadian-Cancer-Statistics-2014--EN.pdf 
[2] Early Breast Cancer Trialists’ Collaborative Group. Polychemotherapy for early breast cancer: an overview of the randomised trials. Lancet. 1998; 352: 930-942. http://dx.doi.org/10.1016/S0140-6736(98)03301-7

[3] Mamounas EP, Bryant J, Lembersky B, et al. Paclitaxel after Doxorubicin plus Cyclophosphamide as Adjuvant Chemotherapy for Node-Positive Breast Cancer: Results from NSABP B-28. J Clin Oncol. 2005; 23 (16): 3686-3696. http://dx.doi.org/10.1200/JCO.2005.10.517

[4] Henderson IC, Berry DA, Demetri GD, et al. Improved Outcomes From Adding Sequential Paclitaxel but Not From Escalating Doxorubicin Dose in an Adjuvant Chemotherapy Regimen for Patients With Node-Positive Primary Breast Cancer. J Clin Oncol. 2003; 21(6): 976-983. http://dx.doi.org/10.1200/JCO.2003.02.063

[5] Urbone A, Gilewski T, Dang CT, et al. Cytokinetics. Cancer Medicine, 5th ed. Edited by Holland JF, Frei E, Bast RC, et al. Baltimore, MD: Williams and Wilkins; 2000. p491-519.

[6] Citron ML, Berry DA, Cirrincione C, et al. Randomized Trial of Dose-Dense Versus Conventionally Scheduled and Sequential Versus Concurrent Combination Chemotherapy as Postoperative Adjuvant Treatment of Node-Positive Primary Breast Cancer: First Report of Intergroup Trial C9741/Cancer and Leukemia Group B Trial 9741. J Clin Oncol. 2003 ; 21 (8): $1431-1439$. PMid:12668651. http://dx.doi.org/10.1200/JCO.2003.09.081

[7] Hudis C, Seidman A, Baselga J, et al. Sequential Dose-Dense Doxorubicin, Paclitaxel, and Cyclophosphamide for Resectable High-Risk Breast Cancer: Feasibility and Efficacy. J Clin Oncol. 1999; 17 (1): 93-100. PMid:10458222.

[8] Hudis C, Fornier M, Riccio L, et al. 5-year result of dose-intensive sequential adjuvant chemotherapy for women with high-risk node-positive breast cancer: a phase II study. J Clin Oncol. 1999; 17 (4): 1118. PMid:10561169.

[9] Fornier MN, Seidman AD, Theodoulou M, et al. Doxorubicin followed by sequential paclitaxel and cyclophosphamide vs. concurrent paclitaxel and cyclophosphamide: 5- year results of a phase II randomized trial of adjuvant dose-dense chemotherapy for women with node-positive breast carcinoma. Clin Cancer Res. 2001; 7 (12): 3934-3941. PMid:11751485.

[10] Bonadonna G, Zambetti M, Valagussa P. Sequential or alternating doxorubicin and CMF regimens in breast cancer with more than three positive nodes. JAMA. 1995; 273: 542-547. PMid:7837388. http://dx.doi.org/10.1001/jama.1995.03520310040027

[11] Dang C, Fornier M, Sugarman S, et al. The Safety of Dose-Dense Doxorubicin and Cyclophosphamide Followed by Paclitaxel With Trastuzumab in HER-2/neu Overexpressed/Amplified Breast Cancer. J Clin Oncol. 2008; 26 (8): 1216-1222. http://dx.doi.org/10.1200/JCO.2007.12.0733

[12] Morris PG, Dickler M, McArthur HL, et al. Dose-Dense Adjuvant Doxorubicin and Cyclophosphamide Is Not Associated With Frequent Short-Term Changes in Left Ventricular Ejection Fraction. J Clin Oncol. 2009; 27 (36): 6117-6123. PMid:19901120. http://dx.doi.org/10.1200/JCO.2008.20.2952

[13] Baldini E, Gardin G, Giannessi PG, et al. Accelerated versus standard cyclophosphamide, epirubicin and 5-fluorouracil or cyclophosphamide, methotrexate and 5-fluorouracil: a randomized phase III trial in locally advanced breast cancer. Ann Oncol. 2003; 14 (2): 227-232. http://dx.doi.org/10.1093/annonc/mdg069

[14] Venturini M, Del Mastro L, Aitini E, et al. Dose-dense adjuvant chemo-therapy in early breast cancer patients: results from a randomized trial. J Natl Cancer Inst. 2005; 97 (23): 1724-33. PMid:16333028. http://dx.doi.org/10.1093/jnci/dji398 